\title{
GRACE: a prototype for the Gran Sasso Air Cherenkov Experiment
}

\author{
D.S. Levin a , B.C. Barish ${ }^{\text {f }}$, E. Diehl a , A. Habig ${ }^{\text {b }}$, J. Handel a, M. Kertzman ${ }^{\text {e }}$, S. Mufson ${ }^{\text {b }}$, \\ j. Musser ${ }^{b}$, S. Nutter ${ }^{c}$, G. Sembroski ${ }^{d}$ and G. Tarlé ${ }^{a}$ \\ "Randall Laburatry of Physics, Unitersity of Michigan, Amm Arbor, MI 48109, USA \\ 'Departments of Physics and of Astronomy, Indiana Unic ersity, Bloomington, IN 47405, USA \\ ' Laboratori Nazionali del Gran Sasso dell'INFN, Assergi (L'Aquila) 67010, Italy \\ "Department of Physics, Purdue Unicersity, West Lafayette, IN 47907, USA \\ ' Department of Physics, DePauw Unitersity, Greencastle, IN 46135, USA \\ ${ }^{\prime}$ California Institute of Technology, Pasadena. CA 91125, USA
}

Received 1 June 1992

GRACE is a five element array of air Cherenkov light collectors designed to measure the energy of primary cosmic rays and operate on the terrain over the deep underground muon tracking detector, MACRO, at the Gran Sasso National Laboratory in Ita! . Tite coincidence detection of air Cherenkov photons and deep underground muons p.oduced in extensive air showers will allow an association of the primary energy with the muon events observed inderground. We present detailed Monte Carlo calculations of the expected signal, a complete description of our detectors and iest results of their initial deployment at the University of Michigan Peach Mountain Observatory.

\section{Overview}

In this article we report on a joint research program of Monte Carlo simulations and field tests of a five element array designed to view Cherenkov light in extensive air showers (EAS) in coincidence with deep underground muons observed with the MACRO detector [1] at the Gran Sasso Laboratory. The intensity of Cherenkov light directly reflects the energy of the incoming particle so that a measurement of this light can determine the energy of primary cosmic rays initiating these showers. Our program is an effort to measure the energy of events seen by MACRO and is also a feasibility study for the construction of an expanded, multi-element array covering a large surface area that could operate in coincidence with an underground muon detector. Such an array provides large solid angle coverage of the underground detector and allows a sufficiently high coincidence event rate to access the higher energy regions of the cosmic ray spectrum.

The underlying idea of GRACE entails the deployment of narrow aperture Cherenkov telescopes on the mountain above the subterrancizis labore'ury. These telescopes, configured over a square area about $75 \mathrm{~m}$ on a side, are designed to have a field of view of about $8^{\circ} \times 8^{\circ}$, which is sufficient to accommodate the projection of the MACRO underground detector but little else. Their optical axes are oriented so that they point back to the center of MACRO. In this manner, only cosmic ray shower cores directed at the underground detector (to within plus/minus the half opening angle of the telescope aperture) are likely to trigger any of the light collectors in the surface array. Since our signal will be comprised of cosmic ray air showers in which the accompanying muons could potentially trigger the underground detectur, this "narrow aperture" method effectively eliminates many background events with sufficient intensity to trigger the Cherenkov array, but whose muon trajectories fail to intersect the underground detector.

In this surface/underground application we are mostly interested in primary cosmic rays with sufficient energy to produce muons capable of penetrating to the underground detector. Muons in EAS can traverse a distance $h \mathrm{~km}$ of Water Equivalent ( $\mathrm{km}$ WE) to the underground laboratory when their energy at the surface exceeds the threshold [2]

$E_{\mu}[\mathrm{TeV}]=\left(0.53 \exp ^{-0.4 h}-1\right)$.

The average depth of the MACRO detector is $3.7 \mathrm{~km}$ $\mathrm{WE}$, thus $L_{\mu}=1$.? TeV. This implies that the primary energy defining our lower energy range of interest is a few Tev.

The objectives of GRACE include a determination of the response, trigger efficiency, coincidence rate with the underground detector, background noise, overall reliability and stability, and live time fraction. 
We consider these issues to be of paramount imporfance to the design and operation of a large scale system. Some aspects of proton-air nucleus interaction physics can be addressed by the diata taken jointly with a surface air Cherenkov array and underground muon tracking detectors. Furthermore, we can use the expected high trigger efficiency to calibrate the effective MACRO primary energy threshoid and we can exploit the excellent angular resolution of a Cherenkov array to provide an independent pointing calibration of MACRO.

\subsection{Motiration and objectives}

An underground cosmic ray detector such as MACRO observes high energy muon bundles (a muon bundle is a group of one or more muons) produced in the collisions of primary cosmic rays at the top of the atmosphere. Measurable properties of these bundles are their muitiplicity, lateral separation and angular distribution. These distributions are sensitive to the composition of high energy cosmic rays and features of hadronic interactions such as the transverse momentum distribution and inclusive charged particle production [2] of the primaries in the atmosphere. Underground detectors do not measure the energy of the primary. In order to extract information on the energy dependence of primary composition and hadronic interactions, more information is needed than can be obtained by the underground detector alone.

The combined operation of the EAS array, EASTOP [3], with MACRO at Gran Sasso is an important step in satisfying this requirement. However, the primary energy threshold typically associated with EAS arrays (and for EASTOP [4] in particular the trigger efficiency is $10 \%$ at $\sim 100 \mathrm{TeV}$ ) neglects an important energy region under $100 \mathrm{TeV}$ in which cosmic rays produce muons in MACRO. Because of the steeply declining power law energy spectrum for cosmic rays, a significant fraction of MACRO events occur in this energy band. Implementation of an air Cherenkov photon detector above MACRO is motivated by the need to provide a measurement of primary cosmic ray energies in this interval below the threshold of the EAS detector. In addition, our array provides a complimentary measurement of the primary energy above 100 $\mathrm{TeV}$ where the EAS array is sensitive.

We now focus on the practical aspects and potential performance of a small prototype Cherenkov array that could operate in concert with MACRO, and also serve as a model unit cell in an expanded array. The proposed active area of the prototype will be large enough to allow us to collect, in a reasonable operating time, a sufficient number of events to study muon multiplicity and lateral pair separation in the energy region of 10-100 TeV (see section 4 for a discussion of event

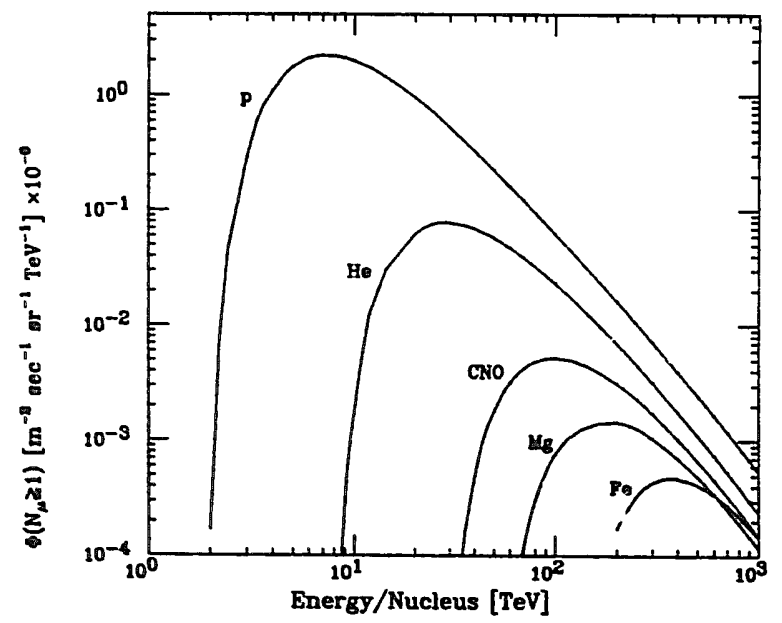

Fig. 1. The rates for a vertically incident primary nucleus to yield at least 1 muon at the average MACRO depth of $\mathbf{3 7 0 0}$ MWE.

rates). In this domain, virtually ali events in which at least one muon reaches the detecter depth are initiated by a cosmic ray proton. To illustrate this point, we show in fig. 1 the differential rates, $\Phi\left(N_{\mu} \geq 1, A, E\right)$, for primary nuclei of mass $A$ and energy $E$ to produce at least one muon at the MACRO mean depth. We assume elemental abundances in accord with a "light" cosmic ray composition; [5] these abundances are extrapolated from low energy measurements to the $\mathrm{TeV}$ region. The curves plotted in fig. 1 are the product of the Poisson probability to obtain at least one muon at depth $h=3700 \mathrm{MWE}$ from a progenitor nucleus at incident zenith angle $\theta$ and the primary mass spectra, i.e.,

$$
\begin{aligned}
\Phi\left(N_{\mu} \geq 1\right)= & \left(1-\exp ^{-\bar{N}_{\mu}(A, E, \theta=0, h=3700)}\right) \\
& \times K(A) E^{-2.71},
\end{aligned}
$$

where the average number of muons in an event, $\bar{N}_{\mu}(A, E, \theta, h)$, is a parameterization of deep underground average muon yield in air showers [6]. Below about $100 \mathrm{TeV}$ we see that MACRO is mostly sensitive to muons produced by cosmic ray proton interactions. Heavier elements have insufficient energy per nucleon to produce muons that can penetrate to the detector depth and, below $100 \mathrm{TeV}$ protons are the most abundant species. We conclude that multiple muon studies in this energy domain focus mainly on features of the proton-air interaction in a poorly explored energy domain, namely, inclusive charged particle cross sections and transverse momenta distributions in the fragmentation region.

The use of Cherenkov radiation as a means to determine the energy and lateral distribution of EAS dates from 1957 and has been employed in numerous experiments since. Such arrays exist for example, at Haverah Park (University of Durham) [7], Yakutsk 
(Former Soviet Union) [8], and Adelaide (Australia). [9]. These arrays generally operate in conjunction with electron counters (plastic or liquid scintillators) to aid in locating the core of the EAS. A single initial attempt to record Cherenkov light in coincidence with an underground muon detector [10] has been successful, albeit with very low statistics.

Detection of Cherenkov light in these experiments has been accomplished with photomultiplier tubes (PMTs). We note a recent effort to detect ultraviolet (UV) photons employing UV sensitive TMAE gas in a proportional chamber [11]. While there are uncertainties associated with atmospheric absorption at these wavelengths, this technique appears promising. TMAE gas is not sensitive to optical wavelengths and operation during moonlight and daytime has been demonstrated.

\section{Cherenkov radiation in EAS}

\subsection{Essential features}

Cherenkov radiation in EAS is produced by relativistic electrons and positrons, and to a lesser extent muons, with $\beta>1 / n(h, \lambda)$, where $\beta$ is the velocity of the particle in units of the velocity of light in vacuum, and $n(h, \lambda)$ is the altitude $(h)$ and wavelength $(\lambda)$ dependent index of refraction of air. Cherenkov photon emission occurs when: $E_{\mathrm{c}}(h, \lambda) \geq m c^{2} / \sqrt{1-n^{-2}}$, where $E_{c}$ the critical energy, and $m$ is the particle mass. For electrons, $E_{\mathrm{c}}=21 \mathrm{MeV}$ at sea level, and increases to $35 \mathrm{MeV}$ at an altitude of $8 \mathrm{~km}$. The half opening angle, $\theta$, of the Cherenkov wavefront is obtained from $\cos \theta=1 /(\beta n(h, \lambda))$. At sea level, $\theta$ is about $1.3^{\circ}$. The number of Cherenkov photons radiated by a charged particle per unit path, 1 , per unit wavelength is given by [12]

$$
\frac{\mathrm{d}^{2} N}{\mathrm{~d} l \mathrm{~d} \lambda}=\frac{2 \pi e^{2}}{\lambda^{2}}\left[1-\frac{1}{(\beta n(h, \lambda))^{2}}\right] \text {. }
$$

There are practical limits to the wavelengths which are detectable at given altitudes. The altitude for our application is $2000 \mathrm{~m}$ above sea level (a.s.l.). A short wavelength limit (250-280 nm) for air Cherenkov radiation is introduced by atmospheric ozone. Although ozone concentration peaks at high altitudes $(20 \mathrm{~km}$ a.s.l.), Cherenkov piotons are radiated predominantly at lower elevations characteristic of maximum shower development $(5-10 \mathrm{~km})$ and are still somewhat attenuated. The ozone abundance [13] below $8 \mathrm{~km}$ a.s.l. is $0.004 \mathrm{~cm} \mathrm{~atm} \mathrm{~km}^{-1}$, while the absorption coefficient at $250 \mathrm{~nm}$ is $300 \mathrm{~cm}^{-1} \mathrm{~atm}^{-1}$. The atmospheric attenuation length due to ozone is just over $800 \mathrm{~m}$ and therefore UV light produced in the upper regions of the

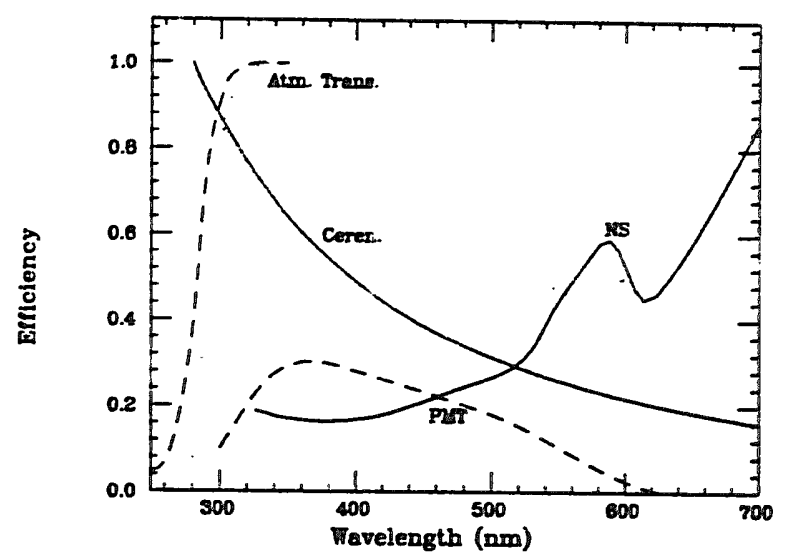

Fig. 2. Response curves and spectra. "Atm. Trans" dashed and dotted lines are transmissions of atmospheric ozone from $2-5 \mathrm{~km}$ and 2-10 $\mathrm{km}$ elevations, respectively; The dashed line, "PMT", is the typical response of an EMI Corporation D642, $20 \mathrm{~cm}$ diameter phototube; "NS" is the characteristic night sky spectrum; "Ceren" is the unnormalized $\lambda^{-2}$ characteristic Cherenkov spectrum; The night sky and Cherenkov spectra are representative of their general shapes and do not indicate relative fluxes.

cascade will be greatly attenuated. The useful longer wavelengths are limited by the background light from the night sky which rises sharply above $600 \mathrm{~nm}$. In addition, the sensitivity of our bi-alkali PMTs becomes vanishingly small above this wavelength.

Fig. 2 shows the spectra of Cherenkov light, a characteristic night sky [14], atmospheric transmittance, and the response curve of our PMTs. The scale for the Cherenkov curve relative to the night sky is arbitrary; the curves indicate that the PMT sensitivity diminishes in the region where the night sky rapidly increases, and that the low end of the Cherenkov spectrum, where light production is greatest, is cut off by atmospheric transmission.

The yield of Cherenkov photons produced in the EAS reflects the energy of the incident nucleus. To evaluate the signal from a single primary particle observed with a ground based detector, we must account for all Cherenkov photons emitted along the path of all charged particles with energy $E>E_{\mathrm{c}}$ and at all depths of the shower. We can express this signal as the number of photoelectrons in a detector of projected area $A(\theta, \phi)$ in the direction $\theta, \phi$, with a wavelength $(\lambda)$ dependent response function $F(\lambda)$,located a distance $s$ from the shower axis, at ar, observation height $\boldsymbol{h}_{0}$

$$
\begin{aligned}
& R\left(E_{\mathrm{p}}, h_{0}, s\right) \\
& =\int_{\Omega} \int_{h_{0}}^{h_{\mathrm{int}}} \int_{\lambda_{\min }}^{\lambda_{\max }} \frac{\mathrm{d} P\left(E_{\mathrm{p}}, h, s, \theta, \phi\right)}{\mathrm{d} h} \frac{\mathrm{d}^{2} N(h, \lambda)}{\mathrm{d} l \mathrm{~d} \lambda} \\
& \quad \times A(\theta, \phi) F(\lambda) T\left(\lambda, \sec \theta, h-h_{0}\right) \Delta l \mathrm{~d} \lambda \mathrm{d} h \mathrm{~d} \Omega .
\end{aligned}
$$


Here $\mathrm{d} P\left(E_{\mathrm{p}}, h, s, \theta, \phi\right) / \mathrm{d} h$ is the differential number, per unit area, per steradian of charged particles in a cascade initiated by a primary proton of energy $E_{\mathrm{p}}$; $h_{\text {int }}$ is the interaction height of the primary particle; $\lambda_{\min }$ and $\lambda_{\max }$ define the useful spectral window determined by the transmission properties of the optics and the atmosphere; $\Delta l(h)$ is the characteristic path length of a relativistic charged particle; and $T(\lambda, \sec \theta, h-$ $h_{0}$ ) is the wavelength dependent atmospheric transmission between production height $h$ and the observer height, $h_{0}$. The angles $\theta$ and $\phi$ define the direction of the light emitting particle in a polar coordinate system in which the upward $z$ axis is negative. The solid angle integration is over the telescope aperture.

The lateral spread of the Cherenkov light on the ground depends on several factors. The first contribution is due to the transverse momenta of secondary neutral pions produced in the first few interactions. Fluctuations in the interaction depth combined with transverse momenta serve to broaden the lateral distribution. Another factor that contributes to lateral separation is multiple Coulomb scattering of the individual electrons in the cascade. Finally, there is a separation of oppositely charged particles in the geomagnetic field. While all these factors combine to spread Cherenkov photons over an extensive area (a scale diameter is on the order of $2-3 \mathrm{~km}$ for several TeV primaries), most of the light is concentrated in a region within a typical distance of $300 \mathrm{~m}$ from the shower core.

\subsection{Monte Carlo simulations}

There is a long history of calculations of Cherenkov photon production in air showers. These range from analytical methods [15] to Monte Carlo techniques of varying degrees of complexity [16-21]. We have evaluated the expected Cherenkov signal by means of a sophisticated three-dimensional Monte Carlo [22] program in which the factors in the integrand above are modeled in detail. From these simulations we can extract the lateral distribution of photons, the energy resolution and sensitivity of the detector array, the lowest energy thresholds commensurate with efficient coincidence detection in MACRO and the photoelectron temporal distribution. A knowledge of the arrival time profile of the leading edge of the Cherenkov wavefront is essential for an accurate measurement of the direction of the shower core.

The Monte Carlo program makes use of the UNICAS cascade program algorithm [23] which specifies primary particle interaction and the production of secondaries. The routine commences with injection of the primary nucleus into the atmosphere. The depth of injection corresponds to a residual atmosphere of $7 \mathrm{~g}$ $\mathrm{cm}^{-2}$. The particle is followed until it interacts and produces secondaries. Each secondary, whose energy

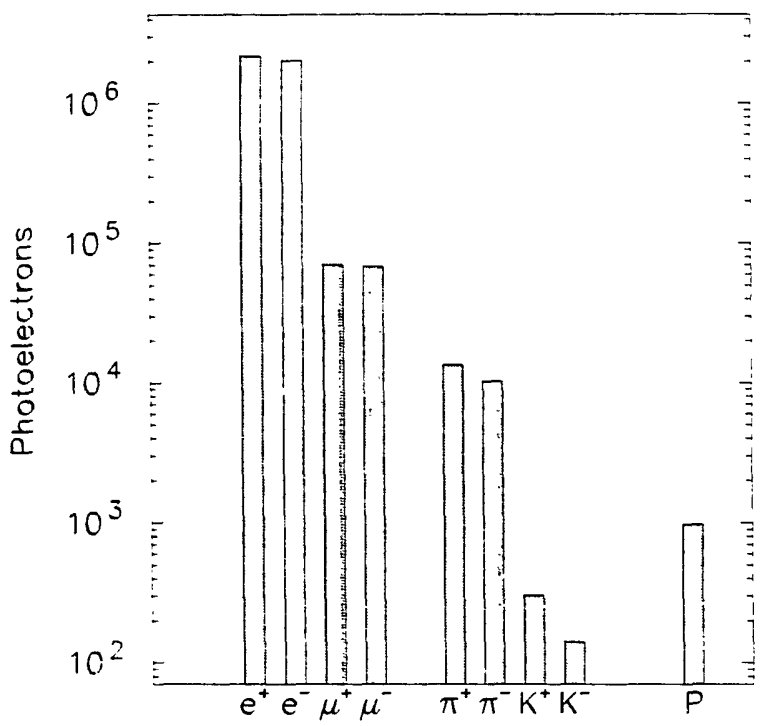

Fig. 3. Numbers of photoelectrons produced by various types of charged particles in the EAS cascade initiated by a vertically incident $10 \mathrm{TeV}$ proton.

exceeds the Cherenkov threshold, is tracked through the atmosphere and Eaith's magnetic field until it either interacts, or decays. Charged particles are propagated in straight line segments corresponding to 0.2 radiation lengths. The endpoint of each segment is redirected to an extent dictated by multiple Coulomb scattering and geomagnetic deflection. The Cherenkov photons are then uniformly radiated between the beginning and ends of these segments and propagated to the observer elevation. The conversion to "detected photons", hereafter referred to as photoelectrons, occurs after the routine accounts for atmospheric attenuation, angular acceptance of the telescope aperture and the combined response of the PMT and optics. Photoelectrons are recorded in the Monte Carlo output with their arrival time, direction and spatial location. We have run Monte Carlo simulations for incident cosmic ray protons over an energy range of 5-100 $\mathrm{TeV}$, and a zenith angle range from $0^{\circ}$ to $33^{\circ}$.

In fig. 3 we show the contribution of different particle types to the Cherenkov pulse for a vertically incident, $10 \mathrm{TeV}$ proton. Electrons and positrons dominate the signal, but the muon component is important. Muons can radiate Cherenkov light all the way down to the observer and can create local fluctuations in intensity. These fluctuations can be identified as clumpiness in certain regions of fig. 4 where the photoelectron distribution on the ground resulting from a single vertically incident $5 \mathrm{TeV}$ proton. Each point in the figure corresponds to a photoelectron that would be seen by one of our detectors located within a grid having a $\mathbf{6 . 2}$ $\mathrm{m}$ spacing.

The expected number of photoclectrons in a single telescope as a function of radial distance from the 


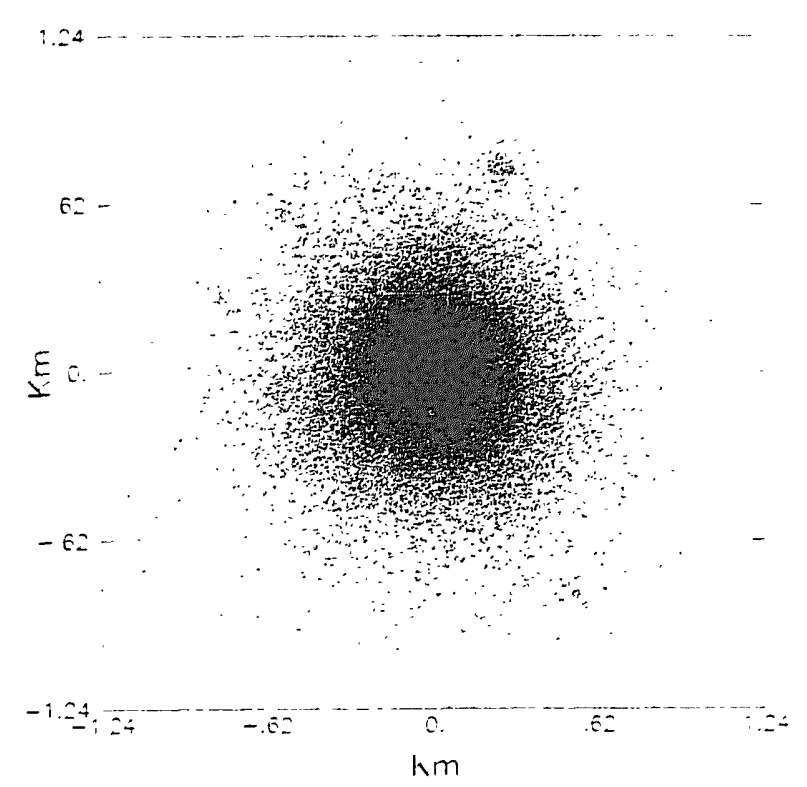

Fig. 4. Light yield from a single $5 \mathrm{TeV}$ proton at vertical incidence.

shower col (for vertically incident protons) is shown in fig. 5 for proton energies of $5,10,20,30,50,75$ and $100 \mathrm{TeV}$ (curves from bottom to top respectively). The horizontal line represents a minimum bias trigger level set al twice the expected night sky light fluctuation (see section 2.3). Two important features are that the lateral distribution tends to scale with energy and that within $100 \mathrm{~m}$ from the core the number of photoelec-

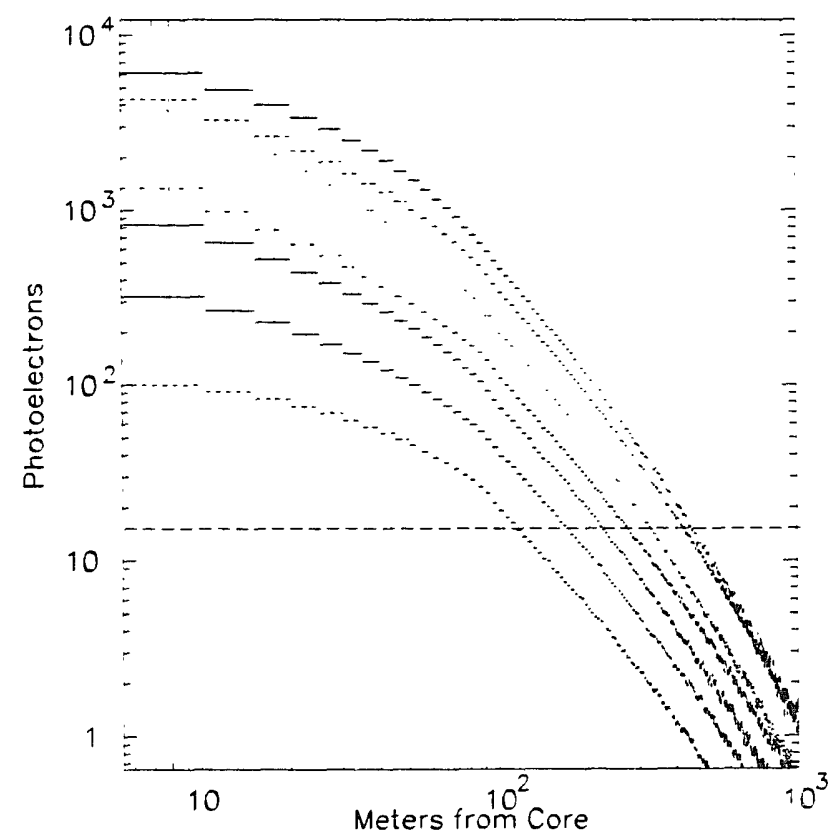

Fig. 5. Average photoelectron distributions for 5, 10, 20, 30 , 50,75 and $100 \mathrm{TeV}$ vertically incident proton (from bottom to top respectively). These curves represent the average number of photoelectrons produced in ten Monte Carlo simulated events at each energy. trons produced by a $10 \mathrm{TeV}$ proton exceeds 50 . This signal should be easily detectable above the expected I ackground of about 7 photoelectrons for a moonless night sky.

In order to estimate the energy of the primary from pulse height measurements the position of the shower core must be known. In practice, the core location may be obtained from the arrival direction of muons observed in MACRO. The $1.0^{\circ}$ random error (from scattering in the overburden) in arrival direction [24] translates into a $\sim 20 \mathrm{~m}$ error in core location at the surface. Furthermore, the non-Gaussian distribution of scattering angles can occasionally introduce a larger uncertainty in core location. However, when multiple telescopes observe the same event, we can check the shower core location by requiring that the core direction (as determined by the array) and the observed pulse heights are consistent with the core location determined by MACRO.

We note also that the absolute energy measurement is subject to systematic uncertainties in the Monte Carlo due to uncertainties in the hadronic interaction, transverse momenta distribution of collision products and deviations (from the assumed idealized model) of the atmospheric profile. The atmospheric transmission may exhibit large fluctuations, particularly in the blue wavelengths. In practice we can measure, while we acquire data, the apparent brightness of a reference star in the same region of sky exposed to our detectors.

The relative energy resolution may be estimated from fig. 6, which shows the lateral distribution from

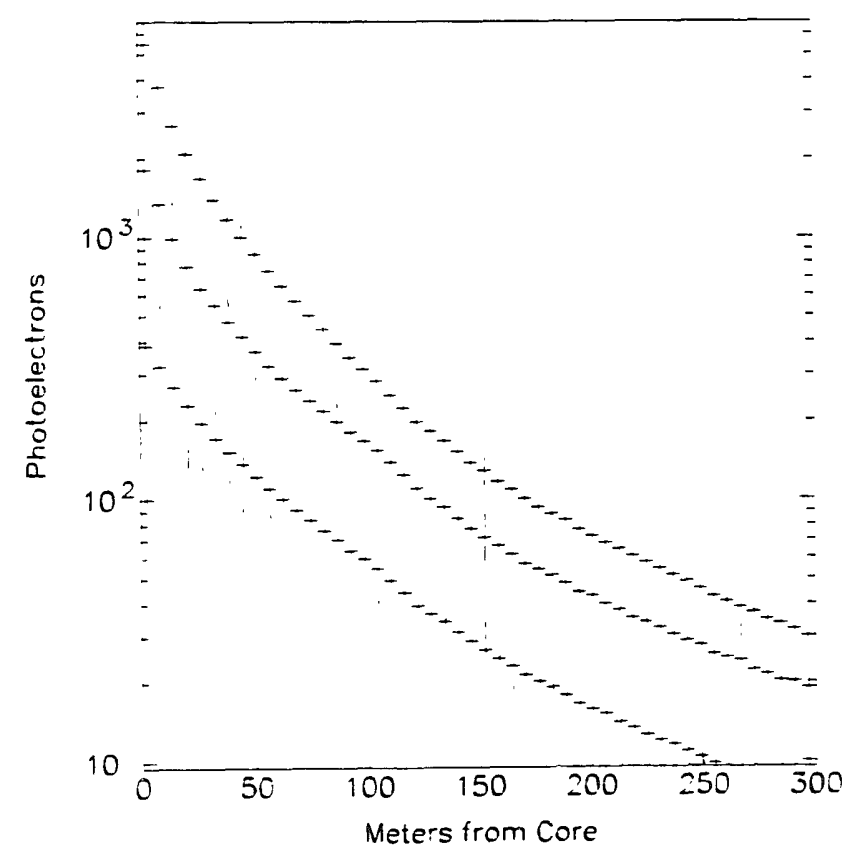

Fig. 6. Lateral distributions from IU, 30 and $50 \mathrm{TeV}$ vertically incident protons with the expected event-by-event statistical error. 
10. 30 and $50 \mathrm{TeV}$ verticallv incident protons with the expected statistical error from a single shower. There are three contributions to these statistical uncertainties. In a single event the amount of light observed at a fixed radius varies randomly around the shower core. This fluciuation arises from structure in the core caused by multiple neutral pions produced early in the cas. cade. Each neutral pion can initiate a separate cascade. Spatial fluctuations in a single event are also caused by muons whose trajectory passes near a telescope. This uncertainty is reduced as more detectors participate in the measurement. A second source of statistical uncertainty results from shower-to-shower fluctuations, such as variations in the initial interaction height. Such uncertainties are non-reducible. The least significant uncertainty at these energies is due to photoelectron statistics. For a factor of three increase in primary energy, between 10 and $30 \mathrm{TeV}$, we estimate the intrinsic resolution to be about three standard deviations of photoelectron number for measurements made at $100 \mathrm{~m}$ from the core. When pulse heights are obtained much nearer to the core, the resolution is reduced by the larger fluctuations that result from the core structure. Conversely, measurements much more distant from the core suffer from photoelectron fluctuations. Furthermore, the resolution may be degraded by the systematic, time-dependent errors associated with atmospheric transmission and climatic conditions.

When the array views showers arriving from nonzero zenith angles, we expect some increase in attenuation as well as broadening of the radial distribution due to the $\sec \theta$ dependence on path. Two processes contribute to these effects. The projectile particle traverses more matter in the atmosphere and, on average, interacts higher. This greater interaction height broadens the lateral distribution of the electromagnetic cascade. In addition, Cherenkov light at the observer's elevation is attenuated by the longer path. We have investigated the angular variation of the radial distribution for 5 and $20 \mathrm{TeV}$ events for a range of zenith angles, and in particular for $33^{\circ}$, the angle of the center of the prototype array will make with respect to MACRO. The radial photoelectron distribution is evaluated about the shower core in the plane orthogonal to the shower direction. This is necessary because the projection of the light spot on flat terrain is a slightly distorted ellipse, whose ratio of minor to major axis lengths is approximately equal to the cosine of the incident shower angle. The distortion is introduced by geomagnetic effects. We also assume that the shower direction is colinear with the optical axes. This assumption introduces little error when the average wavefront direction is off axis by less than $4^{\circ}$ because the detector's aperture extends to this angle, as fig. 7 confirms. Fig. 8 shows these radial distributions for $10 \mathrm{TeV}$ protons at 0,15 and $33^{\circ}$. Whereas nonzero zenith

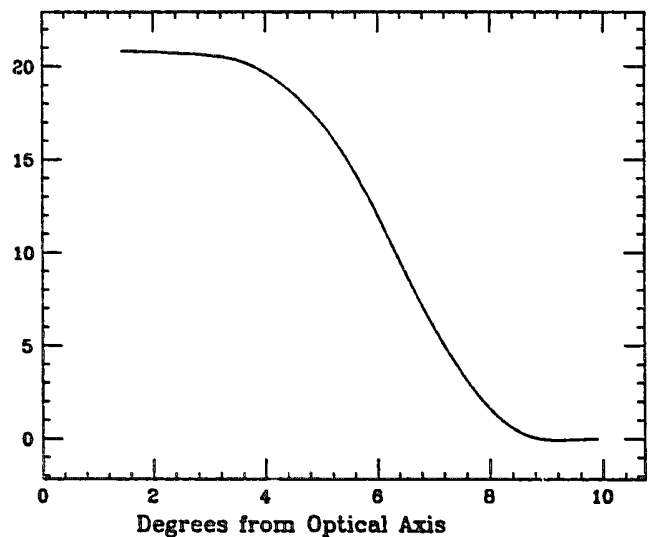

Fig. 7. Geometrical telescope acceptance vs zenith angle for bins of $\cos \theta=0.04$.

angles have larger fluctuations, the number of photoelectrons at a distance of $100 \mathrm{~m}$ from the shower core changes very little. Other systematic errors result from uncertainty in the hadronic interaction employed in the Monte Carlo or from the detector's optical response (the latter is estimated to be much smaller than the statistical uncertainty). These errors introduce an overall shift in absolute energy calibration, but should not significantly alter the relative energy resolution. In fig. 9 we plot the ratios of the average number of photoelectrons produced in 50 and $20 \mathrm{TeV}$ showers. This curve is reasonably flat in the region greater than $50 \mathrm{~m}$ from the shower core, and indicates that the the Cherenkov light flux, when measured away from the core, tends to scale with energy. Fig. 10 shows the

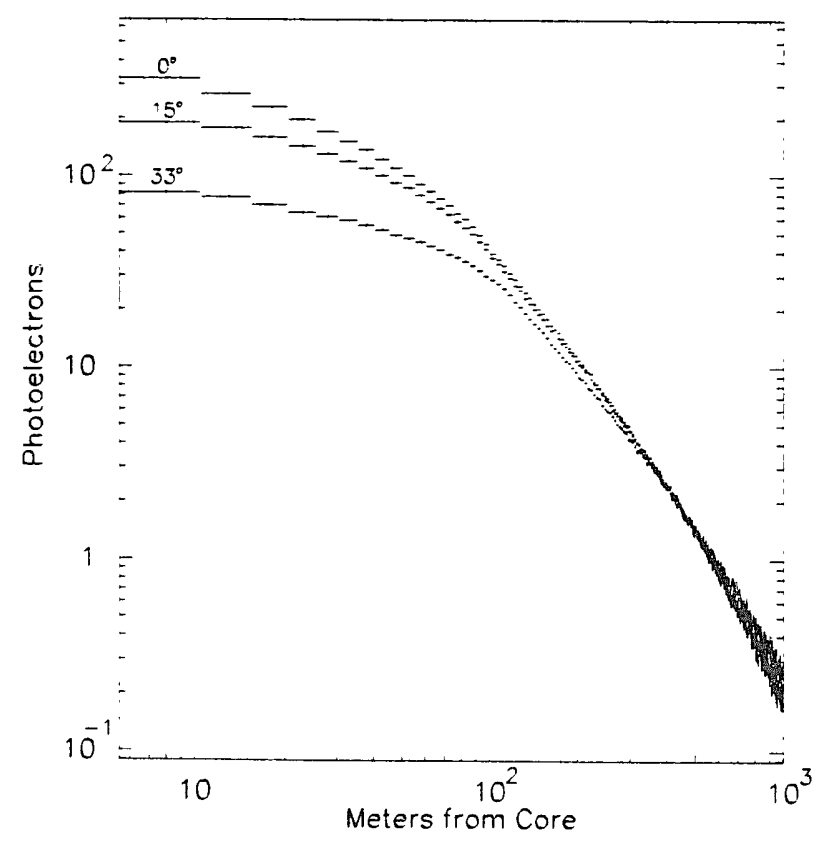

Fig. 8. Average radial photoelectron distribution for $10 \mathrm{TeV}$ protons for zenith angles of $0^{\circ}, 15^{\circ}$, and $33^{\circ}$. 


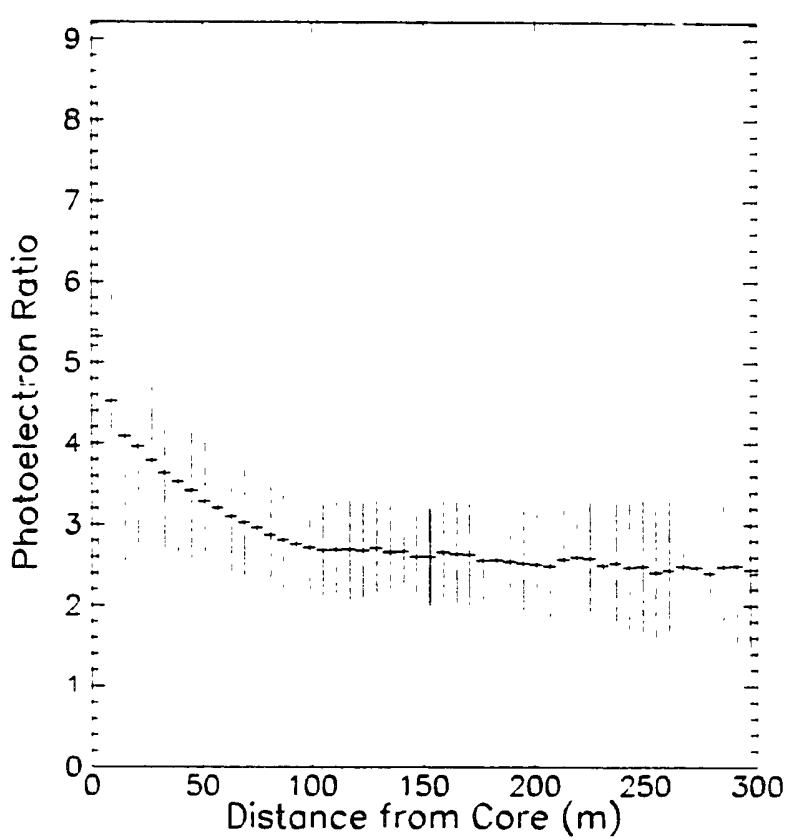

Fig. 9. The ratio of the average number of photoelectrons produced by 50 and $20 \mathrm{TeV}$ protons. distribution of photoelectrons at increasing distances from the core. Although there is more light very close to the center $(\sim 25 \mathrm{~m})$, the structure in the core broadens the distribution and makes energy determination near the core difficult. Figs. 6 and 10 taken together indicate that optimal energy resolution is best at about $100 \mathrm{~m}$ from the core.

\subsubsection{Shower direction and angular distribution}

Cherenkov light that is emitted in the cascade has a characteristic opening angle that depends upon the local index of refraction of air. The net opening angle of the wavefront is the superposition of all such wavefronts in the shower. Coulomb scattering and geomagnetic separation deflect the trajectories of the charged particles resulting in a broadened angular distribution as seen in fig. 11. This graph shows the angular deviation of "photoelectrons" relative to the telescope's optical axis $80 \mathrm{~m}$ from the shower core. In the above context "photoelectrons" refers to photons which have passed PMT quantum efficiency selection in the Monte Carlo, but not geometrical selection. From the geomet-
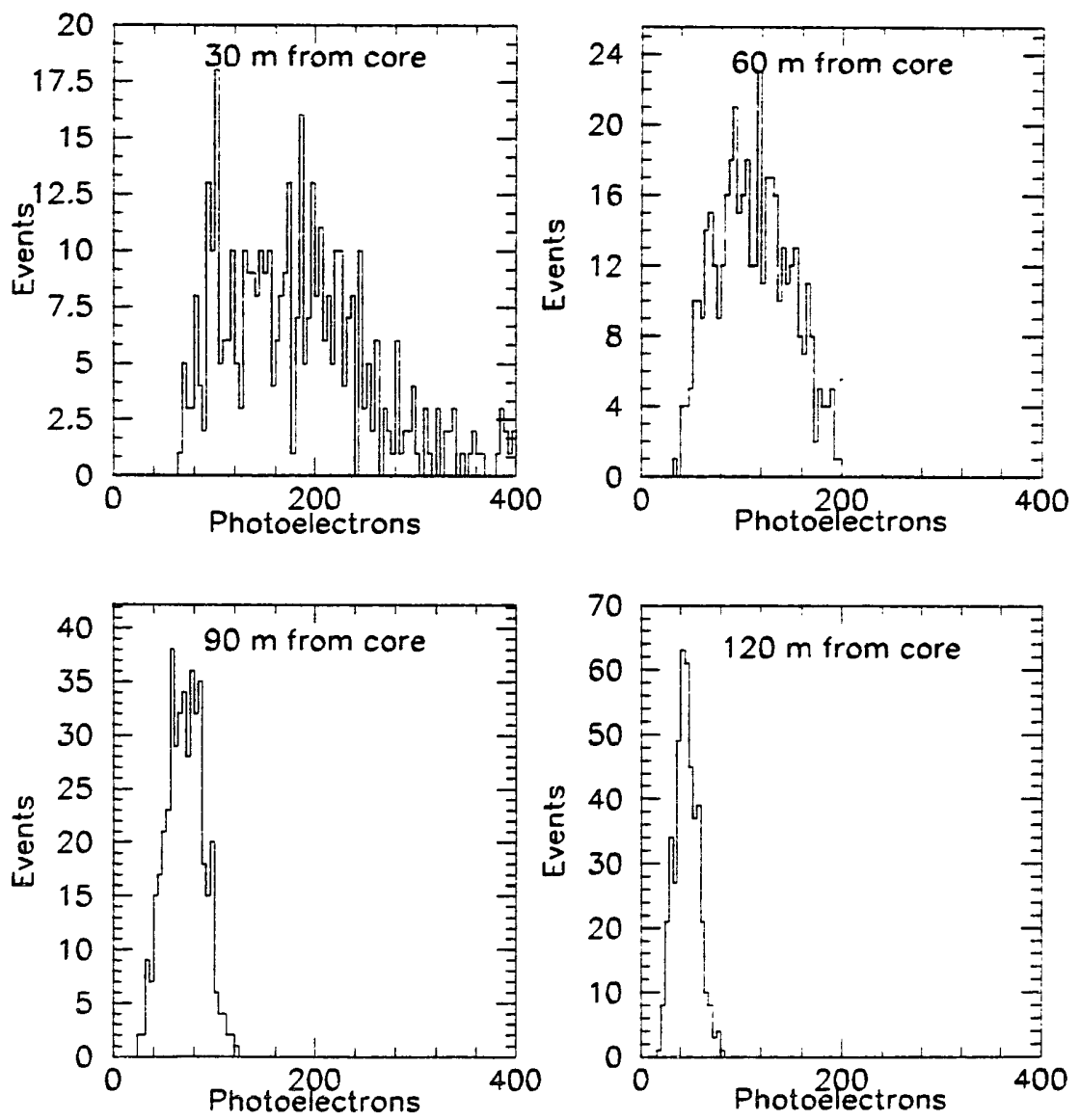

Fig. 10. The distribution of photoelectrons at increasing distances from the air shower core. 


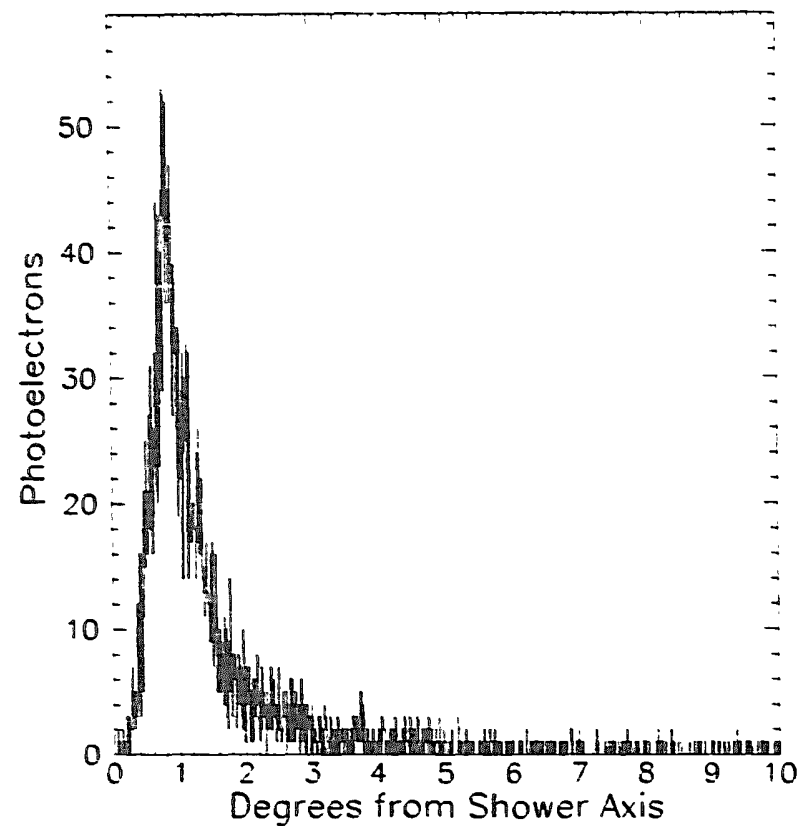

Fig. 11. Angular distribution of Cherenkov photoelectrons in a $10 \mathrm{TeV}$ proton shower incident at $15^{\circ}$ at $80 \pm 6 \mathrm{~m}$ from the shower core. The $x$ axis is the angle of the detected photon's direction with respect to the telescope axis aligned with the core.

rical response, fig. 7, we see that a very small amount of light would be rejected by the aperture.

In fig. 12, we show the time of arrival vs distance from the shower core of the Cherenkov light front for $15^{\circ}$ and for $33^{\circ}$ incidence. In order to measure shower
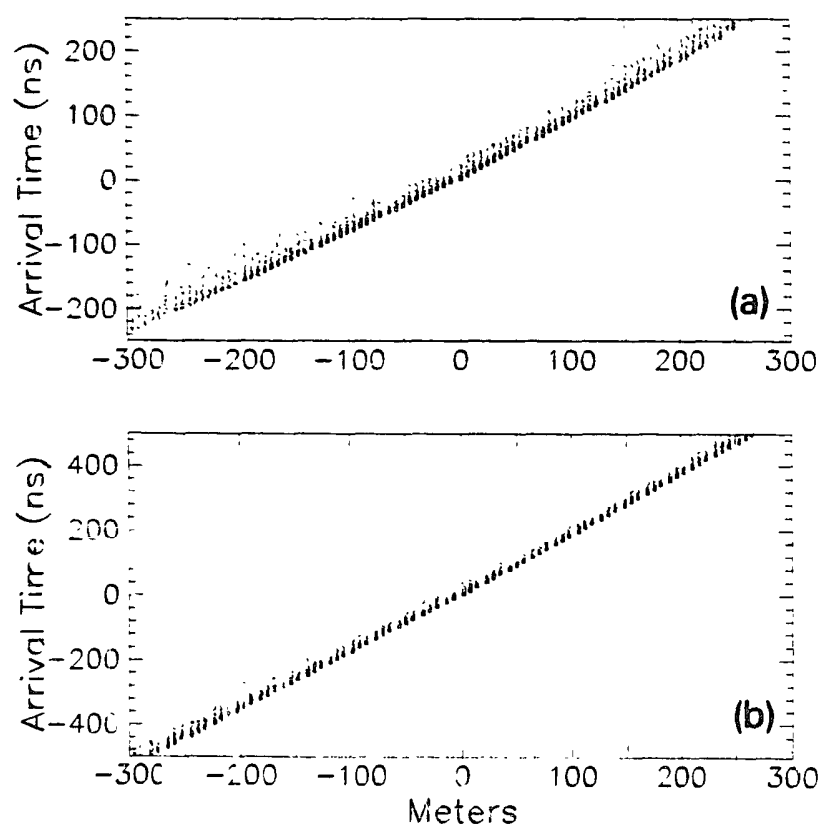

Fig. 12. Time space distribution of the Cherenkov pulse for (a) $15^{\circ}$ and (b) $33^{\circ}$ incident $10 \mathrm{TeV}$ protons. direction, we require multiple precise timing along the light front. The time of arrival also has a characteristic jitter on the order of $5 \mathrm{~ns}$ at $100 \mathrm{~m}$ from the shower core. This spread is largely attributable to the presence of Cherenkov light from nearby muons that precedes the main light front (not resolvable in fig. 12). If we consider that the leading edge of Cherenkov light arrives from a maximum height of about $5 \mathrm{~km}$ relative to the observer (for a $\sim 50 \mathrm{TeV}$ proton), we find that the muon component arrives earlier than the the photon component by about $4 \mathrm{~ns}$. This is consistent with the Monte Carlo result. This time spread implies an error of less than $1^{\circ}$ in determination of arrival direction from a single three telescope stereo measurement with a $100 \mathrm{~m}$ baseline. Since our angular resolution is better than $1^{\circ}$, GRACE can perform a pointing calibration of the MACRO detector. Multiple measurements can reduce the systematic error in MACRO tracking to $0.1^{\circ}$ by this method.

\subsection{Estimation of background from night sky light}

The dominant source of background (on moonless nights) to the Cherenkov signal will be photoelectron fluctuations produced by the quasi-constant background of starlight. The principal limitation imposed by the presence of this ambient light is on the permissable gain at which the telescope PMTs may be operated.

The background noise may be estimated by considering the typical flux of photons integrated over the sensitive range of the PMT from about $300-600 \mathrm{~nm}$. This flux [25], $\Phi_{\mathrm{B}}$, is about $6 \times 10^{11}$ photons $\mathrm{m}^{-2} \mathrm{sr}^{-1}$ $\mathrm{s}^{-1}$ for a dark night sky. The background noise is assumed to be the statistical fluctuation of the photocathode occupancy, i.e.,the average number of photoelectrons ejected from the photocathode over the resolving time, $\tau$, of the PMT. The background noise is calculated from

$\delta N_{\mathrm{pe}} \sim \sqrt{\Phi_{\mathrm{B}} A \Omega \epsilon \tau}$

where $A \times \Omega$ is the acceptance and $\epsilon$ is the spectrum averaged photocathode quantum efficiency. For our telescopes, the typical fluctuation is expested to be approximately 7 photoelectrons. Ambient light trom nearby towns, scattered by dust in the atmosphere above the array, may also contribute to this background.

\section{The prototype array}

\subsection{Instrument design}

The mechanical and optical design of the Cherenkov detector is optimized for maximum light collection 
efficiency within the spectral range $300-500 \mathrm{~nm}$, miniunum background from starlight, atmospheric scintillation, static discharges, mechanical rigidity, reproducible pointing ability and overall robustness for deployment in a somewhat hostile climate and uneven terrain. Special features include heating elements to dissipate condensation on optical surfaces and a provision for closing the aperture during daylight. When the sun crosses the field of view, the focused sunlight on the phototube can vaporize the photocathode and melt the giass envelope.

The principal detector elements are a 36 in. focal length, 32 in. diameter parabolic mirror (manufactured at Purduc University) of the type incorporated in the Haleakala Gamma Ray Observatory [26] and an 8 in. EMI D642 hemispherical ghototube supported at the mirror's focal plane. The photocathode quantum efficiency is highest between $350-400 \mathrm{~nm}$ (peaking near $400 \mathrm{~nm}$ at $\sim 25 \%$ ). The opening angle will be large enough to subtend the entire MACRO detector in the backward field of view. The aperture function, expressed as the acceptance for constant intervals of $\cos \theta$, is shown in fig. 7. The geometrical acceptance is approximately constant to $4^{\circ}$ before falling off. This geometrical response is such that the entire photocathode is illuminated by a Cherenkov wavefront from an EAS whose core points to MACRO but is located over $80 \mathrm{~m}$ away from from the telescope.

\subsubsection{PMT characteristics}

We have studied the PMT response in a combined pulsed and continuous background mode, i.e., under light conditions roughly simulating Cherenkov detection with a very luminous night sky. Our primary concerns are to maintain PMT currents at non-destructive levels (no more than a tens of $\mu \mathrm{A}$ ), and to reduce distortion of the pulse height measurement due to the presence of a background induced current. The result of an initial test indicates that loss of gain is likely when the PMT is continuously subjected to a high dc background light level. We found that our PMTs can operate with a constant dc current of $80 \mu \mathrm{A}$ for at left $240 \mathrm{~h}$ without measurable loss of gain. After nearly 600 $h$ of exposure, the gain dropped by almost $50 \%$. When the background was turned off, there is a partial recovery of gain. This gain history is shown in fig. 13. We regard this test as conservative and thus are not worried by the drop in gain by the 24th day. The $80 \mu \mathrm{A}$ background that we used in the laboratory is twice the background current induced by the local night sky. In practice PMTs are not continuously exposed for more than $8 \mathrm{~h}$. Lastly, we note that a change in gain is easily measurable in our apparatus with light emitting diodes (LEDs) placed in each telescope. We do not expect long term degradation of pulse height resolution.

We have monitored the signal distortion for fast $(10$

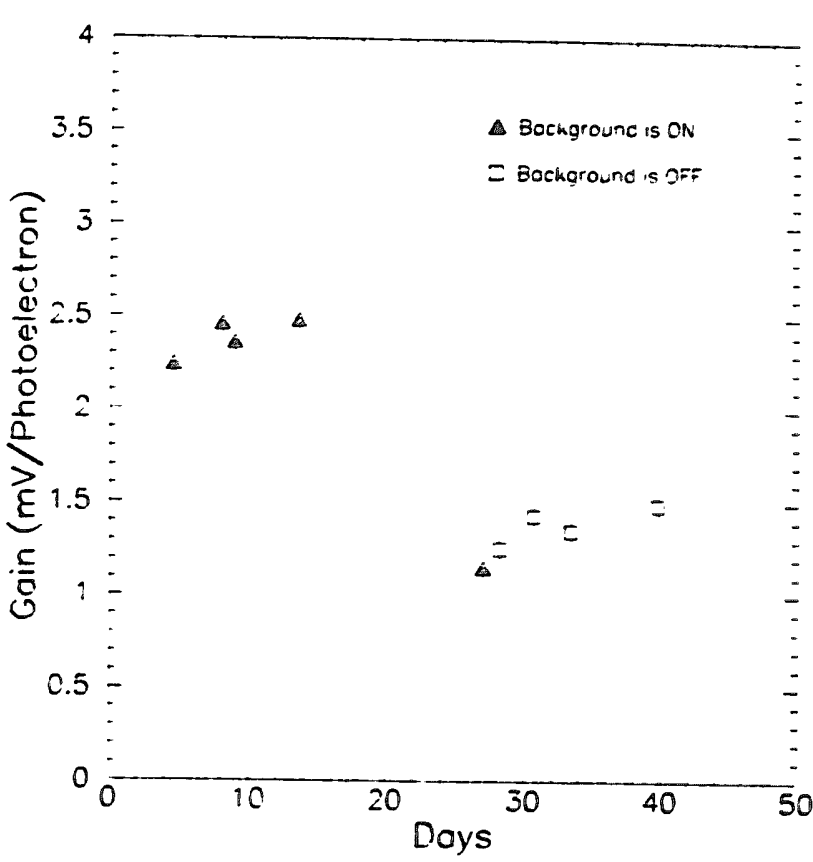

Fig. 13. PMT gain degradation over time when exposed to constant background source. The photocurrent is about $80 \mu \mathrm{A}$.

ns FWHM) LED induced signais with a dc LED background. This distortion is often seen as an increase of pulse height with respect to the pulsed source operating without the background LED. It results from and redistribution of voltages on the PMT divider chain whose effect is to increase the first dynode stage voltage while decreasing the voltage drop at the final stages. We can reduce this distortion by maintaining a base current at least 10 times higher than background induced anode currents and through voltage regulation of the last dynode stages. Also, the PMT operating voltage is selected well below the point where severe distortion occurs. A typical operating voltage for our tubes will be between 1000 and $1200 \mathrm{~V}$. In fig. 14 we show the PMT gain curves obtained from a fast (15 ns FWHM) pulsed LED with and without a continuous background. We find that up to nearly $1300 \mathrm{~V}$, little distortion is introduced to the pulse height. A typical gain at this voltage is about $0.25 \mathrm{mV} /$ photoelectron. A $5 \mathrm{TeV}$ proton induced shower, which yields $\sim 20$ photoelectrons at $80 \mathrm{~m}$. from the core, is expected to produce about a $5 \mathrm{mV}$ pulse.

We have also investigated spatial nonuniformity in the photocathode. In our application, light rays reflected from the parabolic mirror converge across the entire face of the I'MT from angles ranging from $0^{\circ}$ to about $24^{\circ}$ with respect to the optical axis. For a fixed incident (pulsed) light flux uniformly illuminating the photocathode, we find the angular dependence of the pulse height to vary by less than $5 \%$ from $0^{\circ}$ to $30^{\circ}$. 


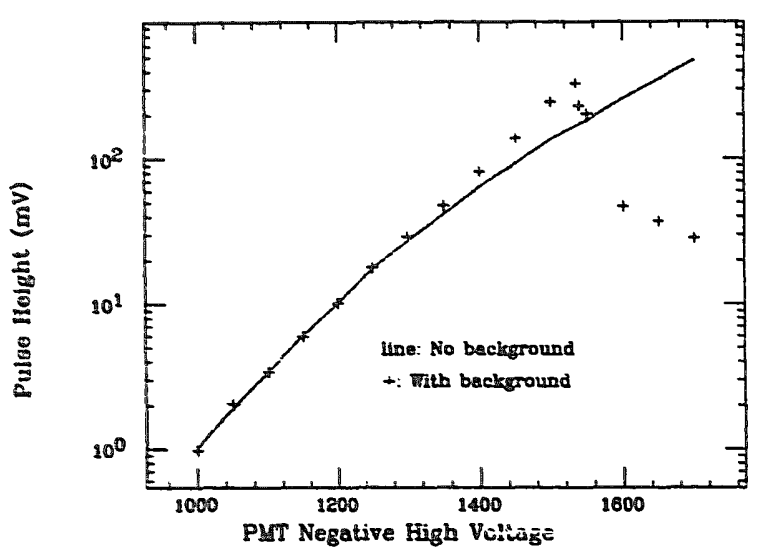

Fig. 14. EMII ihototube pulse height versus voltage for a fast pulsed LED yielding about 80 photoelectrons in the PMT. The data points are taken with one pulsed LED and one constant background LED. The dc anode current induced by the backgroand is $80 \mu \mathrm{A}$.

\subsubsection{Telescope enclosure and mount}

The detector enclosure, fig. 15 , is a double-walled cylinder manufactured from sheet aluminum, framed by cast aluminum rings at each end which are separated by wood spacers. Polyurethane foam insulation fills the gap formed by the two walls and gives the structure additional mechanical rigidity. The mirror resides at one end of the cylinder and is oriented along the axis. pointing towards the opening. The opposite end is enclosed by a UV transparent plexiglass bubbile cover (not shown) mounted over a quick release $\mathrm{O}$-ring seal. The phototube and base electronics are housed in a PVC cannister supported by three lateral aluminum struts secured to the main cylinder.

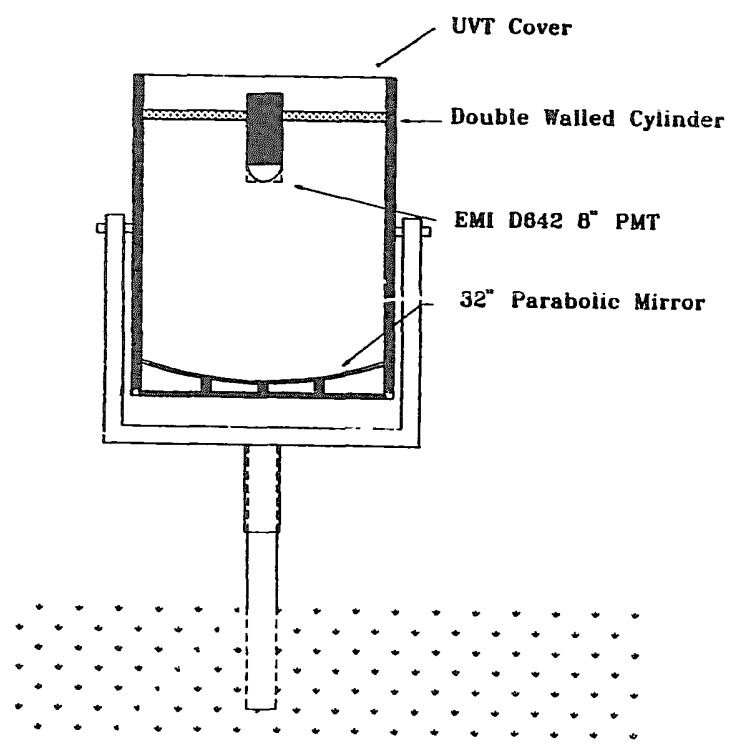

Fig. 15. Diagram of main features of the Cherenkov light collectors.
The mounting for the Cherenkov light buckets consists of 2 in. by 3 in. rectangular cross section steel welded into a "tuning fork" shaped frame. The cylindrical body is designed to pivot about an axis perpendicular to the optical axis and is mounted at the ends of the fork, allowing for polar angle motion. The base of the fork rotates on a vertical axle which joins the fork with the base mounting hardware. This consists of a circular cross section vertical extension of steel terminated in a 1/2 in. thick baseplate. The baseplate can be bolted directly onto a level concrete pad.

A machine engraved dial allows the azimuth to be determined to $\pm 0.25^{\circ}$, and a digital inclinometer provides a $0.1^{\circ}$ precision zenith angle orientation. A side mounted rifle scope is used to reference orientation to known stellar coordinates. The rifle scope is aligned to within $0.2^{\circ}$ of the optical axis.

The opening angle of the aperture is $4^{\circ}$, somewhat larger than the maximum angle subtended by the projection of the MACRO detector at a typical elevation on the Gran Sasso plateau above the laboratory. Due to the elongated shape of MACRO $(72 \times 12 \mathrm{~m})$, the addition of an aperture mask can reduce background events in which the Cherenkov wavefront would generate a trigger but the accompanying muon(s) misses the underground detector.

\subsection{Electronics: front end, triggering and data acquisi- tion}

The data required from each element in the array are a time of arrival and a from the PMT. A time-todigital conversion (TDC) ieast count of 1 ns is sufficient to measure arrival time such that the resolution of the electronics does not contribute significantiy to the error. As mentioned above, the ambient night sky light mandates low gain PMT operation $\left(\sim 10^{5}\right)$, and thus front end amplification is required to produce signal amplitudes required for trigger formation. In order to measure a large dynamic range of pulse heights, we use two sets of ADCs, one with a $20 \mathrm{~dB}$ voltage amplification.

The triggering modes depend somewhat on the exact array configuration and will be determined by field tests. In our basic configuration the five telescopes are arranged in a cross of about $80 \mathrm{~m}$ along the sides. This will permit extensive overlap of the telescopes' regions of sensitivity. In this manner, events whose shower cores fall within an area bounded by a perimeter line connecting the four corners of the cross (internal events) should generally yield multiple (3-5) hits. Events with cores outside the perimeter (external events) should generate 1-3 fold hits. Trigger criteria may be simply defined by a majority of 1 to 5 telescopes over threshold within a 100 ns time window. 
Data acquisition is performed by a personal computer (PC) linked with a CAMAC data acquisition system. With a maximum expected trigger rate of a few $\mathrm{Hz}$, and about 25 bytes of data per event,the acquisition computer must acquire a few Mbytes per day. The PC will also perform various housekeeping tasks such as temperature monitoring and control, event rate monitoring and operation of an automated telescope instrumented with a CCD camera to measure the absolute atmospheric transmission. Presently there is no direct data link between the surface array and the underground laboratory. Data from the independent experimenis must be merged in the offline analysis stream, and correlated by means of accurate event timing. An absolute time for all telescope triggers is recorded by a latchable quartz clock with a $32 \mu$ s least count. It will be synchronized with the Universal Time clock in the MACRO data acquisition system. The precision and stability are such that unambiguous event identification is possible with the MACRO data. The technique for accomplishing this has been previously demonstrated [3].
In addition to recording times and amplitude with TDCs and ADCs, we must monitor sky conditions. Fluctuations in the atmospheric profile (water vapor, dust and pollutants, aerosols and ozone) affect transmission of Cherenkov light. We will monitor night sky transmission with a dedicated PMT instrumented telescope oriented towards a reference star of known brightness. The PMT output will be incorporated into the data stream to provide continuous information on the sky transmission. In addition, a signal rate monitor of the total trigger rate can provide independent feedback on the combined long term (h-d) variability of atmospheric conditions and the electronics gain.

\subsection{Results of field tests at Peach Mountain}

The five prototype Cherenkov telescopes were deployed for a field test in April, 1991 at the Peach Mountain site of the University of Michigan Radio Observatory. This site, 15 miles from the Ann Arbor campus, afforded night skies relatively free of urban light pollution. Unfortunately, springtime climatic con-
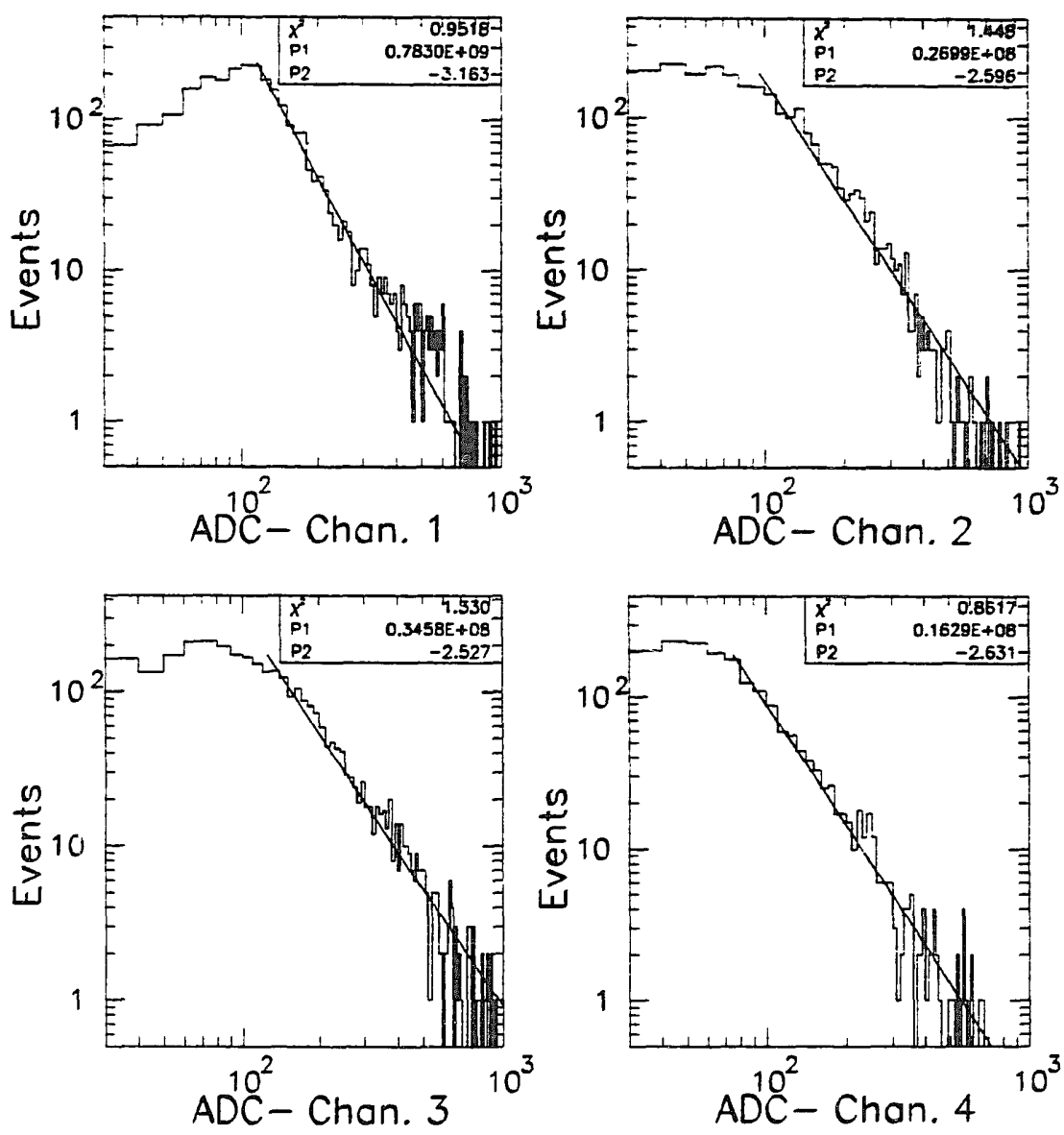

Fig. 16. The pulse height spectra obtained from the four telescopes. We have fitted the pulse height spectra in these plots with a power law having an index given by parameter $\mathrm{P} 2$. The fits have been applied only to data in the the final ADC decade in order to avoid any influence from efficiency effects, which are difficult to evaluate. 
ditions were such that cloudy skies persisted throughout more than $95 \%$ of the available night hours. As a consequence, only a fraction of the data were taken than might otherwise have been the case.

The configuration of the five stations was roughly in the shape of a cross, with typical dimension of $30 \mathrm{~m}$ along one side. A survey of the positions was performed in order to allow measurement of arrival direction from timing information and a knowledge of the baselines. The pointing ability of the telescopes at the time of the test was crude $\left(1^{\circ}-2^{\circ}\right)$ compared to the current, upgraded design. Two orientations of the units were tried: toward the zenith, and toward the star Polaris.

The first observation of Cherenkov signals was made simply by monitoring the PMT outputs, with and without amplification, on a digitizing oscilloscope. Pulses exhibiting a characteristic shape, having a fast rise time and long tail, were seen. Superposition of the signals from 4 telescopes was performed with delay lines and it was possible to observe two, three and four fold coincidences directly on the oscilloscope.

We can estimate the night sky light background in this field test from the dc "baseline" photocurrent. A typical current induced by back rround light was about $40 \mu \mathrm{A}$. For our nominal PMT gain of about $2 \times 10^{4}$, the average occupancy in the rot ghly $20 \mathrm{~ns}$ resolving time was 250 photoelectrons. The level of the background fluctuations was $\sim 16$ photoelectrons.

Event rates were monitored under conditions varying from clear and moonless to partial moon and to completely overcast skies. Clear, dark nights yielded a single telescope event rate of $0.05 \mathrm{~Hz}$. With heavy cloud cover, the rate dropped by over an order of magnitude. The source of light under overcast conditions, in which the cloud cover is $\sim 1 \mathrm{~km}$, is presumably due to muons. Cherenkov light from the more copious electrons and positrons is produced above the clouds. Contrary to our expectations, operation of the telescopes during periods of partial moon light was possible, provided that no rays directly entered the aperture. We intend to assess further and quantize telescope performance with partial moonlight during the Gran Sasso deployment.

Over several hours on consecutive nights, data were acquired on a LeCroy 224912 bit ADC, and LeCroy 2228 TDC ( $0.25 \mathrm{~ns}$ least count). In fig. 16 we have plotted the pulse height spectra obtained from the four telescopes. We have obtained a power law fit to the pulse height spectra in these plots. The fits have been applied only to data in the final ADC decade in order to avoid any influence from efficiency effects, which are difficult to evaluate. We emphasize that these are rough fits, with low statistics, in which no corrections for shower core distance or variations in atmospheric transparency have been applied. Nonetheless, if we

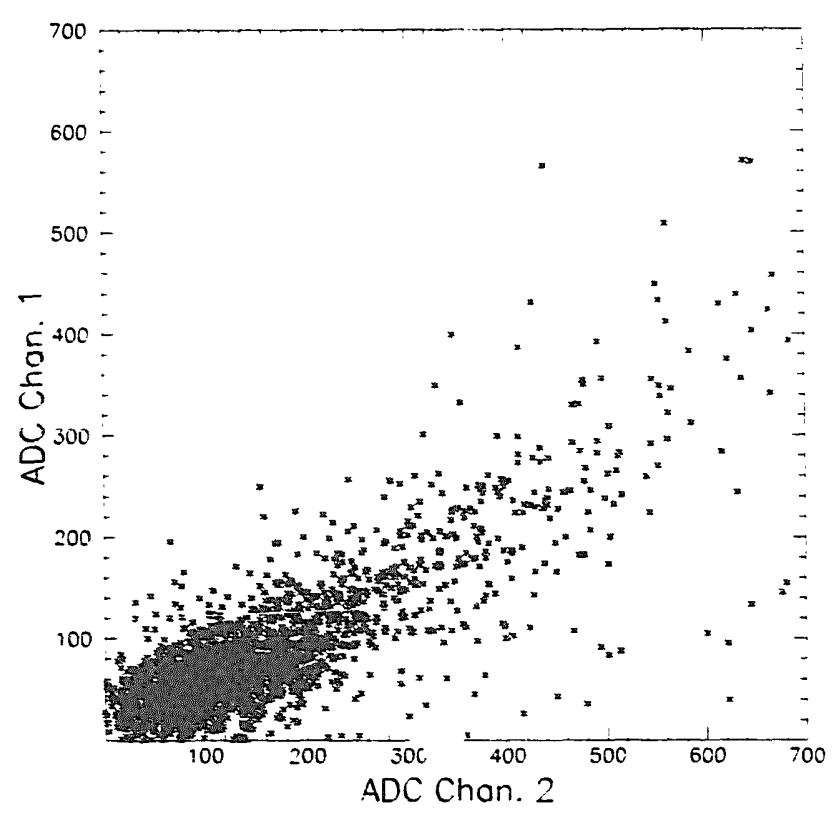

Fig. 17. Scatter plot of pulse heights from two telescopes separated by $30 \mathrm{~m}$.

take the variation of the slope parameter as an indication of the error, these spectra are consistent with the power law index $y \approx-2.7$ of the all-particle spectrum. In fig. 17 we show a plot of the ADCs from two telescopes separated by $30 \mathrm{~m}$ that triggered on the same event. The correlation in pulse heights is evident.

To measure arrival direction, it is first necessary to obtain the TDC offsets. This is accomplished by pointing the telescopes at the zenith. In this orientation, the average time of arrival difference is zero, and the TDC

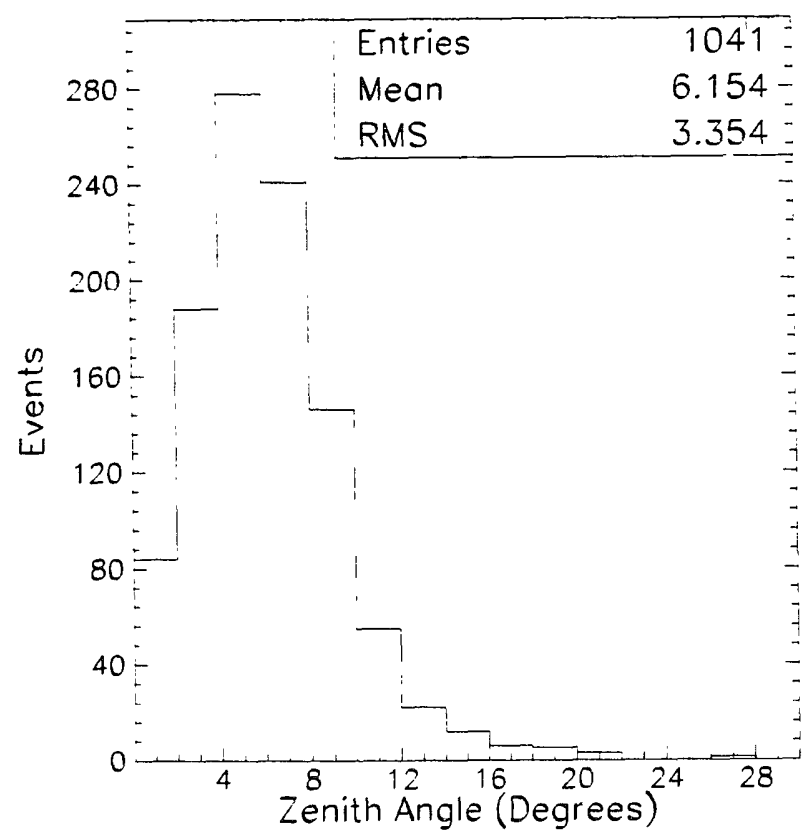

Fig. 18. The zenith angle projection determined from the arrival times for telescopes oriented vertically. 


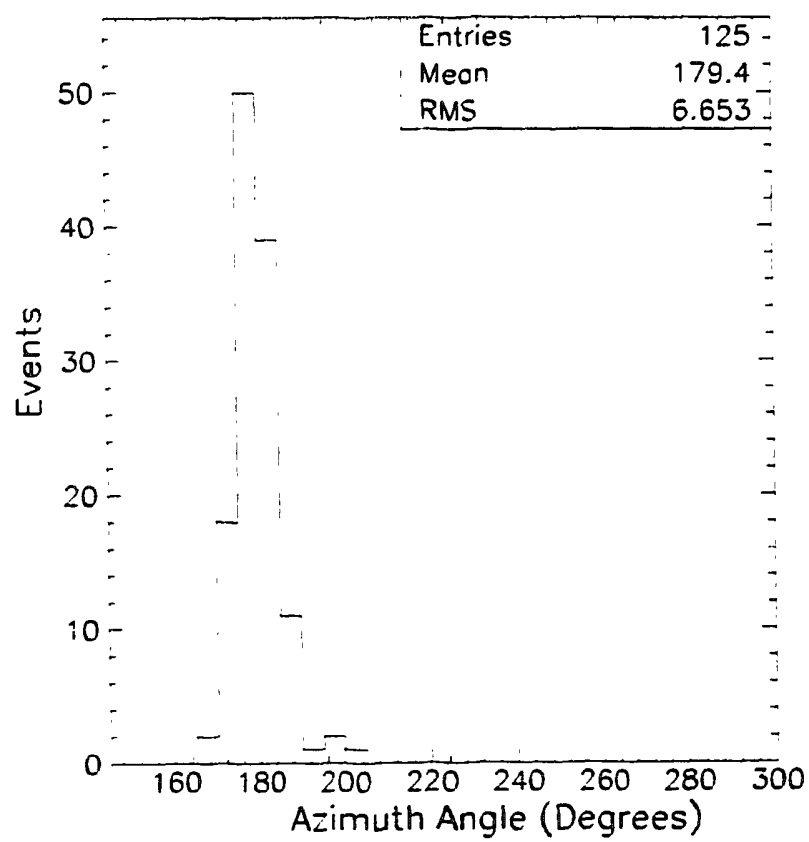

Fig. 19. The reconstructed azimuthal arrival directions. Telescopes are oriented toward the North Star at $42^{\circ}$ zenith angle. offsets are tuned to oblain this result. The zenith angle projection, determined from the arrival times for upward orientation, is shown in fig. 18. The width of the distribution corresponds to the $11^{\circ}$ half-angle aperture used in the field test. The telescopes were then oriented toward the North Star and data were collected. The reconstructed azimuthal arrival directions are shown in fig. 19. A Gaussian fit to the azimuthal arrival direction gives $179.2^{\circ} \pm 4.6^{\circ}\left(x^{2}=0.7\right)$, consistent with geographical north in our test configuration.

We are encouraged by ihese resuits given the paucity of data and relatively crude pointing mechanisms of the telescopes in use at the time. These indicate that measurement of arrival directions with sub-degree precision (over $\sim 100$ events) is possible.

\section{Deployment of the prototype array at Gran Sasso}

The five station prototype array will be located roughly $550 \mathrm{~m}$ south and $350 \mathrm{~m}$ west from the center of the MACRO detector at an elevation of $2000 \mathrm{~m}$ a.s.l.
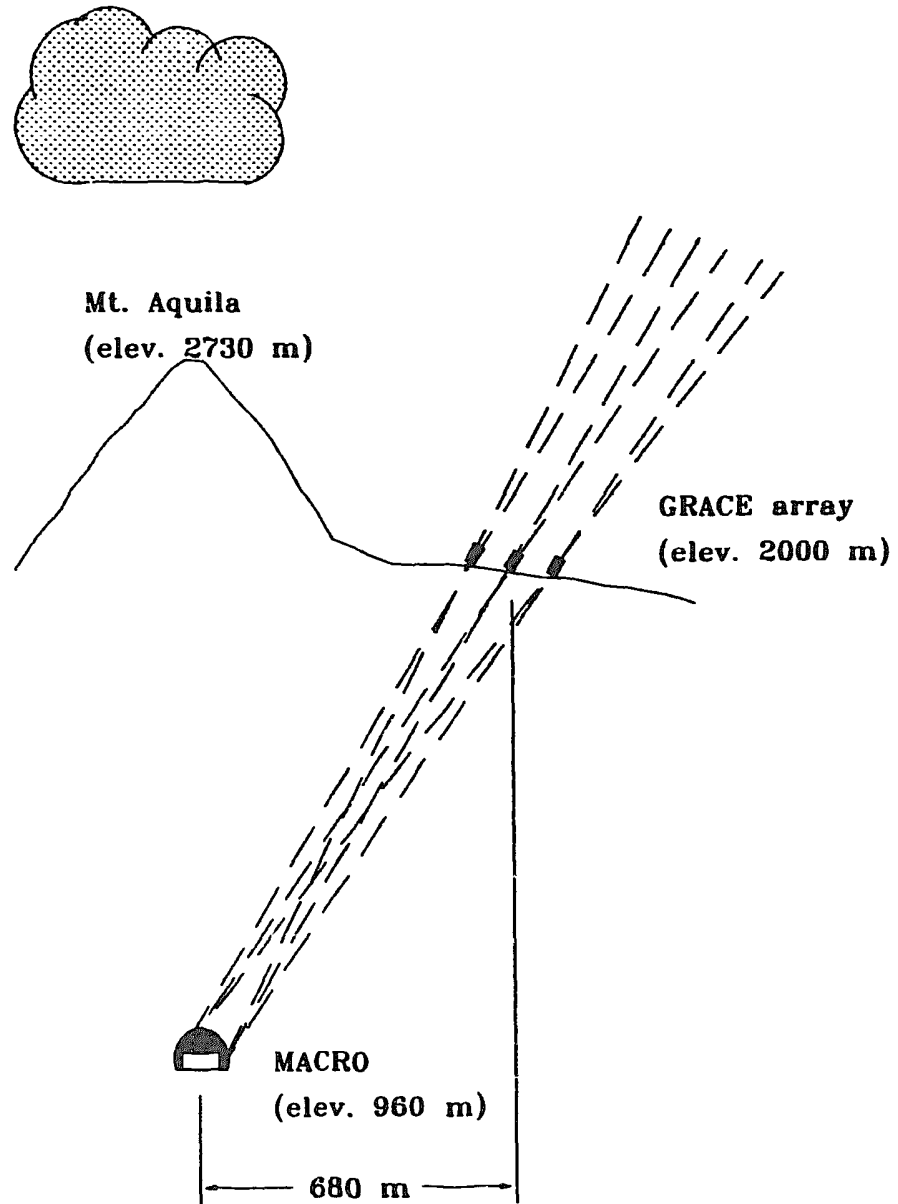
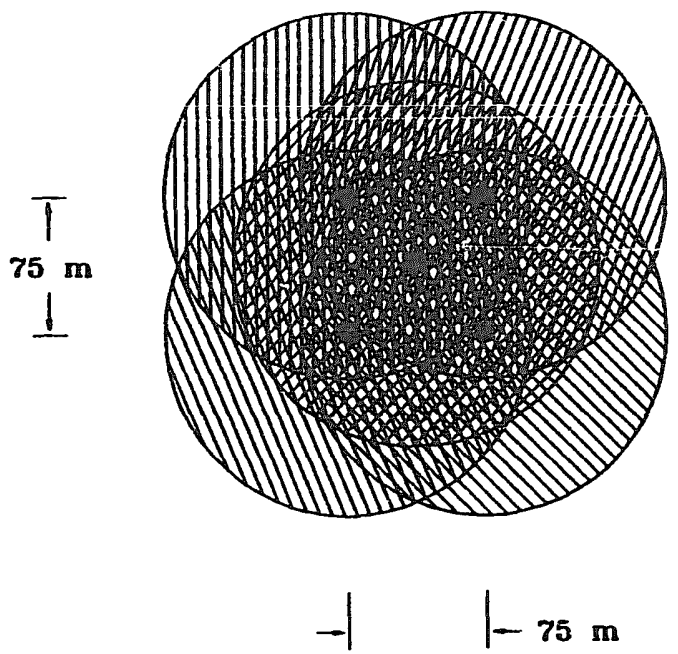

Fig. 20. (a) Profile of proposed telescope array configuration on the mountain above the MACRO detector. (b) Top view of the array. Circles represent the approximate shower core distance range required for the telescopes to trigger on $10 \mathrm{TeV}$ pulses with greater than $90 \%$ efficiency. 
In fig. 20 we show a profile and top view of the arrangement. In the profile view a line from the array center towards $\mathrm{MACRO}$ forms a $33^{\circ}$ zenith angle. The top view indicates ranges of sensitivity of the telescopes to trigger on $10 \mathrm{TeV}$ showers. Cores that pass within a given circle are expected to generate a trigger with greater than $90 \%$ efficiency. About $90 \%$ of all primary protons producing muons at the MACRO detector depth have an energy in excess of $10 \mathrm{TeV}$.

The rate of coincidence events with MACRO can be estimated from the approximate solid angle subtended by the Cherenkov array. We restrict the fiducial region to one in which the full telescope aperture is active. The aperture response function (fig. 7) limits us to arrival directions less than $4^{\circ}$ off the optical axis. For an $80 \mathrm{~m}$ separation between stations, and a $100 \%$ trigger efficiency with respect to MACRO, the fiducial array area will subtend a solid angle through which roughly $0.5 \%-1 \%$ of all muons observed in MACRO.

The fraction of MACRO muon events expected to be intercepted by GRACE is estimated from the azimuthal and zenith angle distributions [27]. With a projected muon detection rate for the complere MACRO detector of approximately $800 / \mathrm{hr}$ [27], we expect to detect several events in coincidence per live hour.

\section{Summary}

We have undertaken experiments to establish the experimental feasibility of operating an air Cherenkov array in temporal coincidence with the MACKO deep underground muon detector. We have designed, constructed and field-lested five prototype light collectors and have conducted a detailed Monte Carlo simulation of Cherenkov photon production in EAS, inclucing a realistic model of our apparatus. The results of the simulations indicate that for most primary nuclei with sufficient energy to produce energetic muons that penetrate to MACRO, our telescopes collect sufficient light to efficiently trigger above a minimum threshold invoked by the ambient night sky light. This threshold, which is twice the expected night sky induced noise of under 10 photoelectrons, is below the signal expected from a $10 \mathrm{TeV}$ proton primary at $100 \mathrm{~m}$ from the shower core.

The calculated rate of coincidence events for our array is on the order of a few per live hour. This will allow us to collect information to assess a curately the performance of the joint system and consider realistic options for an expansion to an extended array covering a significant solid angle with respect to the underground detectors. Our practical, short term goals involve using the Cherenkov array's angular resolution and absolute orientation to conduct a pointing calibra- tion of MACRO. In addition we expect to use initial energy measurements in coincidence events to study deep underground muon multiplicity and lateral distributions in an energy regime for which the primary cosmic ray interaction is reasonably well known to be be a proton on an air target.

\section{Acknowledgemerits}

We are grateful to the directorship and staff of Laboratori Nazionali del Gran Sasso for their cooperation and encouragement. We thank Jim Gaidos at Purdue Jniversity for his ideas on Cherenkov telescope construction, Giulio Auriemma for helpful discussions, the University of Michigan Department of Astronomy for the use of the Peach Mountain facility and Jim Stone at Boston University for supplying us with PMTs. We are especially thankful to Beth Demkowski for her unfailingly cheerful editing assistance.

This work was partially funded by Department of Energy and the University of Michigan Phoenix Memorial Reactor.

\section{References}

[1] M. Calichio et al., Nucl. Instr. and Meth. A264 (1988) 18.

[2] T.K. Gaisser and Todor Stanev, Nucl. Instr. and Meth. A235 (1985) 183.

[3] The MACRO and EASTOP Collaborations, Proc. 20th ICRC, HE4.4-4, Adelaide, 1990, ed. R.J. Protheroe (U. of Adelaide, Adelaidel p. 335.

[4] The EASTOP and MACRO Collaborations, ibid., p. 331.

[5] M.L. Cherry, et al., Phys. Rev. D27 (1983) 1444.

[6] C. Forti et al., Phys. Rev. D42 (1990) 11.

[7] R.T. Hammond et al., Il Nuovo Cimento 1C (1978) 315.

[8] I.M. Kerschenholz et al., Proc 13th ICRC, AS-3, Denver, 1973, ed. R.L. Chasson (U. of Denver, Denver) p. 645.

[9] P.C. Crouch et al., Nucl. Instr. and Meth. 179 (1981) 467.

[10] M. Aglietta et al. (EASTOP and MACRO Collaborations), Proc. of 2nd Int. Workshop on Neutrino Telescops, 1990, Venezia, p. 209.

[11] B. Bartoli et al., Nucl. Instr. and Meth. A302 (1991) 515.

[12] J.V. Jelley, Čerenkov Radiation and Its Applications (Pergar $>$ n, 1958) p. 21.

[13] W.G. Driscol ed., Handbook of Optics (McGraw-Hill, 1978) Sec. 14-1.

[14] H.E. Smith and M. Burbidge, Astrophys. J. 210 (1976) 627.

[15] W. Galbraith and J.V. Jelley, Nature 171 (1953) 349; and V.I. Zatsepin and A.E. Chudakov, Sov. Phys. JETP 15 (6) (1992) 1126.

[16] R.J. Protheroe and J.R. Patterson, J. Phys. G10 (1984) 841.

[17] J.R. Patterson and A.M. Hillas, J. Phys. G9 (1983) 1433. 
[18] T.J.L. McComb and K.E. Turver, Il Nuovo Cimento 5C (2) (1982) 131.

[19] R.J. Protheroe and K.E. Turver, II Nuovo Cimento 51A (2) (1979) 277.

[20] G.J. Smith and K.E. Turver, J. Phys. A6 (1973) L121.

[21] C. Castagncli, M.A. Locci, P. Picchi and G. Verri, Nucl. Phys. B2 (1967) 369.

[22] M.P. Kertzman and G. Sembroski, Proc. 22nd ICRC, OG 10.3-21, Dublin, 1991, ed. D. O'Sullivan (Dublin Inst. for Adv. Studies, Dublin) p. 656.
[23] T. Gaisser, Cosmic Rays and Particle Physics (Cambridge University, 1990), p. 250.

[24] S.P. Ahlen et al. (The MACRO Collaboration), Nucl. Instr. and Meth. (1992) in press.

[25] See ref. [12], p. 219.

[26] A.T. Kenter, Ph.D. Thesis, University of WisconsinMadison (1989).

[27] S.P. Ahlen et al. (The MACRO Collaboration), submitted to Astrophys. J. (1992). 